\title{
WAVELET-BASED WARPING TECHNIQUE FOR MOBILE DEVICES
}

\author{
Ekta Walia $^{1}$ and Vishal Verma ${ }^{2}$ \\ ${ }^{1}$ Department of Computer Science, South Asian University, New Delhi, INDIA \\ wekta@yahoo.com \\ ${ }^{2}$ Department of Computer Science, M. L. N. College, Yamuna Nagar, INDIA \\ me_vishaal@hotmail.com
}

\begin{abstract}
The role of digital images is increasing rapidly in mobile devices. They are used in many applications including virtual tours, virtual reality, e-commerce etc. Such applications synthesize realistic looking novel views of the reference images on mobile devices using the techniques like image-based rendering (IBR). However, with this increasing role of digital images comes the serious issue of processing large images which requires considerable time. Hence, methods to compress these large images are very important. Wavelets are excellent data compression tools that can be used with IBR algorithms to generate the novel views of compressed image data. This paper proposes a framework that uses wavelet-based warping technique to render novel views of compressed images on mobile/ handheld devices. The experiments are performed using Android Development Tools (ADT) which shows the proposed framework gives better results for large images in terms of rendering time.
\end{abstract}

\section{KEYWORDS}

Image-based rendering, 3D image warping, Wavelet image compression, Novel view generation of compressed images on android-based mobile devices.

\section{INTRODUCTION}

For mobile devices with limited screen size, processing of large images takes considerable amount of time. This is where compression techniques come into act. Various compression techniques have been available, but in the past few years, wavelets have shown to be more efficient than many other methods [1]. The power of wavelets is Multi-Resolution Analysis (MRA) which allows representing different levels of detail of images. The Haar wavelet [2] is one of the simplest wavelet transforms which can be used to transform large images into considerably smaller representations that then can be processed on mobile/ handheld devices at higher speeds. This paper proposes a framework to render novel views of compressed images using Haar wavelet based 3D warping technique on mobile devices. Such a framework is particularly useful in visualization of large images on mobile/ handheld devices at interactive rates. The paper is organized as follows: Section 2 gives an overview of Haar wavelet transformation for lossy image compression; Section 3 explores the image-based 3D image warping technique; Section 4 describes the implementation of the proposed framework for mobile devices using Android Development Tools (ADT); Section 5 provides the experimental results and performance comparison; and Section 6 presents the conclusion. 


\section{HAAR WAVELET TRANSFORM FOR IMAGE COMPRESSION}

Although wavelets have their roots in approximation theory and signal processing, they have recently been applied to many problems in computer graphics like image editing, image compression, animation, global illumination etc [3]. Over the past few years, various waveletbased image compression schemes like Discrete Cosine Transform (DCT) [4], Haar transform [2], Daubechies transform [5] [6] etc. are available, each having their own representation and optimization procedures. Among these techniques, the Haar transform is one that has been mainly used due to its low computing requirements.

An image is a matrix of pixel (or intensity) values; therefore, it can be thought of as two dimensional signals, which change horizontally and vertically. Thus, 2D haar wavelet analysis is performed on images using the concepts of filters. Filters of different cut-off frequencies analyze the image at different scales. Resolution is changed by filtering, the scale is changed by upsampling and down-sampling. First horizontal filtering decomposes the image into two parts, an approximation part (low frequency) and a detail part (high frequency). Then vertical filtering divides the image information into approximation sub-image, which shows the general trend of pixel values; and three detail sub-images, which show the horizontal, vertical and diagonal details or changes in the image. At each level, four sub-images are obtained. Fig. 1 shows haar wavelet transform that divides $\mathrm{N} \times \mathrm{N}$ image into 4 sub-images. Each piece has dimension $(\mathrm{N} / 2) \times(\mathrm{N} / 2)$ and is called Approximation (represented by LL), Horizontal details (represented by HL), Vertical details (represented by LH) and Diagonal details (represented by $\mathrm{HH}$ ) respectively. To get the next level of decomposition, haar wavelet transform is applied to the approximation sub-image.

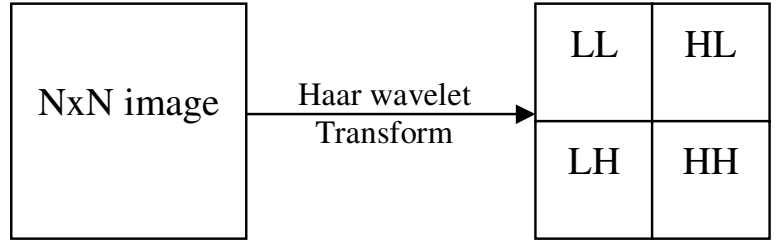

Level 1

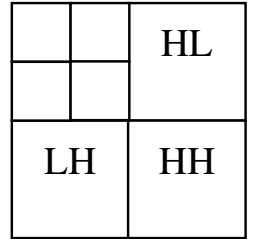

Level 2

Figure 1. Haar wavelet transform of an image

To get a better idea about the implementation of this wavelet in image compression, consider a 512 x 512 pixels grayscale image of the woman (elaine_512.gif) as shown in Fig. 2. By applying the Haar wavelet transform we can represent this image in terms of a low-resolution image and a set of detail coefficients (Fig. 2). The detail coefficients can be used for the reconstruction of the original image.

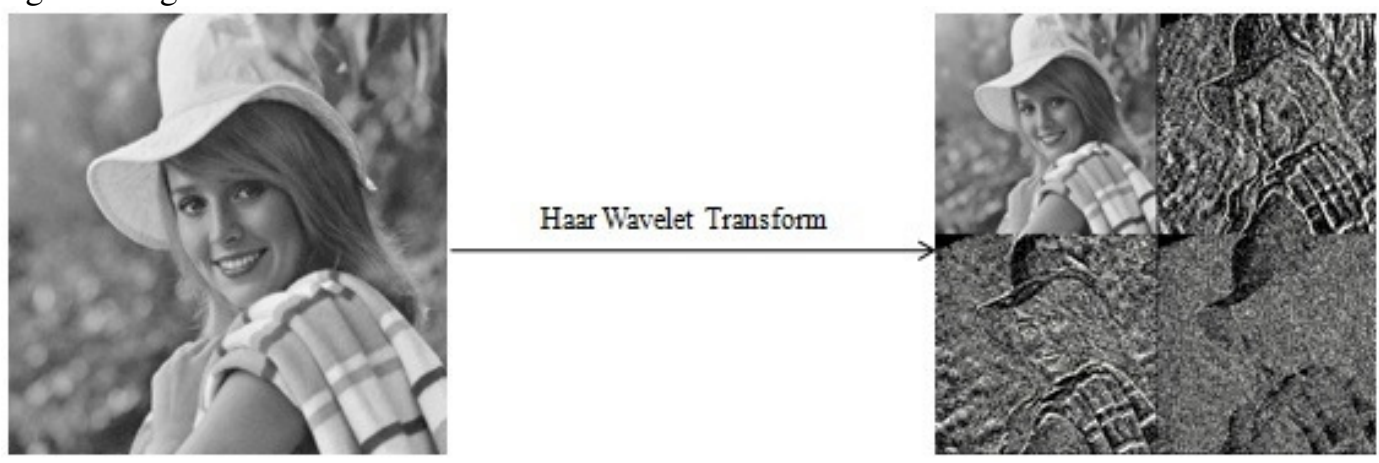

Figure 2. Haar wavelet transform on grayscale image 
In computer graphics, we can use the averaging and differencing technique as the application of Haar wavelet to compress the image. The low-pass (average) filter and high-pass (difference) filter are defined as:

$$
A=(a+b) / 2 \text { and } D=(a-b) / 2
$$

where $\mathrm{a}$ and $\mathrm{b}$ are pixel values of the image. Taking one row at a time, first apply averaging and differencing technique for each pair of pixel values. After treating all rows, apply the same procedure for each column of the image matrix. This produces a matrix containing approximation part (storing the general trend of the image) and detail part (containing most values close to zero). For example, consider the upper left 8 × 8 section of grayscale image in Fig. 2. Fig. 3 shows the resultant matrix by applying averaging and differencing procedure on this matrix.

$$
\left[\begin{array}{llllllll}
190 & 190 & 190 & 190 & 190 & 192 & 192 & 192 \\
190 & 190 & 190 & 190 & 190 & 192 & 192 & 192 \\
190 & 190 & 190 & 190 & 190 & 192 & 192 & 192 \\
190 & 190 & 190 & 190 & 190 & 192 & 192 & 192 \\
190 & 190 & 190 & 190 & 190 & 192 & 192 & 192 \\
190 & 190 & 190 & 190 & 190 & 192 & 192 & 192 \\
190 & 190 & 190 & 190 & 190 & 192 & 192 & 192 \\
190 & 190 & 190 & 190 & 190 & 192 & 192 & 192
\end{array}\right] \quad \text { Transform } \rightarrow\left[\begin{array}{ccccccccc}
190 & 190 & 191 & 192 & \text { । } & 0 & 0 & -1 & 0 \\
190 & 190 & 191 & 192 & 1 & 0 & 0 & -1 & 0 \\
190 & 190 & 191 & 192 & 1 & 0 & 0 & -1 & 0 \\
190 & 190 & 191 & 192 & 1 & 0 & 0 & -1 & 0 \\
- & - & - & - & 1 & - & - & - & - \\
0 & 0 & 0 & 0 & 1 & 0 & 0 & 0 & 0 \\
0 & 0 & 0 & 0 & 1 & 0 & 0 & 0 & 0 \\
0 & 0 & 0 & 0 & 1 & 0 & 0 & 0 & 0 \\
0 & 0 & 0 & 0 & 1 & 0 & 0 & 0 & 0
\end{array}\right]
$$

Figure 3. Haar wavelet transform on image matrix

In images, low frequency (changing slowly over the image) information is usually a lot more than high frequency (quickly changing) information. Due to this, most of the values resulting from the high-pass filter are close to 0 . The more of these values which are close to 0 , the more affectively the image can be compressed.

Grayscale image consists of a single matrix, but for RGB images, there are 3 matrices of same size to represent three colors: red, blue and green. Therefore, we apply Haar wavelet transform on 3 different matrices separately. Fig. 4 shows the approximation part of 512 x512 pixels RGB image (Lena.bmp) after applying one level Haar wavelet transform which is close to original image.

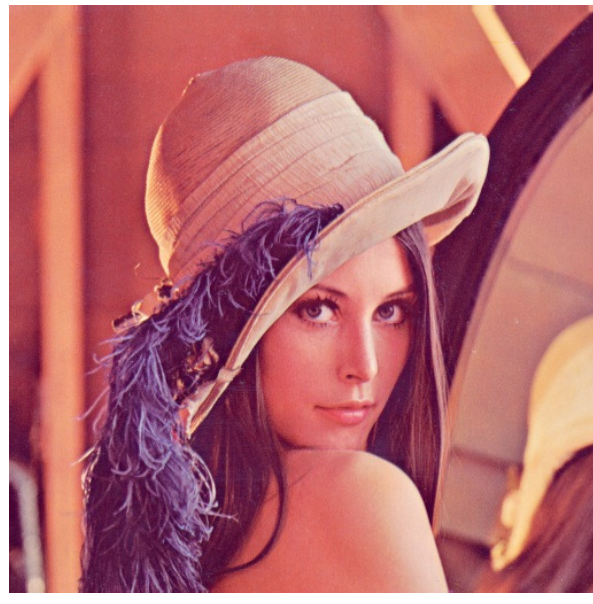

Original Image (512 x 512)

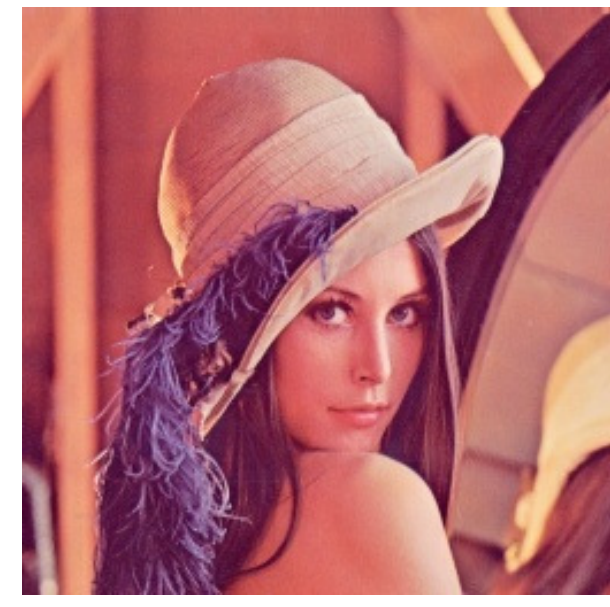

Compressed Image (256 x 256)

Figure 4. Haar wavelet transform to compress RGB image 


\section{3D IMAGE WARPING}

$3 \mathrm{D}$ image warping is an image-based rendering (IBR) algorithm that allows a $2 \mathrm{D}$ reference image to be viewed from a different view position and/ or orientation [7]. The reference image contains color information as well as depth information for each pixel. The processing required for IBR is independent of scene complexity but instead dependent on screen resolution. As such it is especially suited for rendering on low-end mobile devices with small screen size. The central computational component of 3D image warping technique is a mapping function, which maps pixels in the reference images to their coordinates in the target image according to the following equation:

$$
x_{d}=\delta\left(x_{r}\right) P_{d}^{-1}\left(C_{r}-C_{d}\right)+P_{d}^{-1} \mathrm{P}_{\mathrm{r}} x_{r}
$$

where $\boldsymbol{x}_{d}$ is the result of mapping of the point $\boldsymbol{x}_{r}$ on reference image to the desired image, whose centers of projection are $C_{r}$ and $C_{d}$ respectively. $\left(C_{r}-C_{d}\right)$ is a vector between the two centers of projection. $P_{r}$ and $P_{d}$ represent the pinhole camera viewing matrices for reference and desired image respectively. $P_{d}$ is computed each time the user changes orientation or position of camera to generate a novel view of the reference image. The quantity $\delta\left(x_{r}\right)$ is called the generalized disparity for point $x_{r}$ which is inversely proportional to the depth. 3D warping requires that this value to be known for all points in the reference image.

Mapping using 3D Warping equation is not one-to-one. Therefore we must resolve visibility. McMillan describes such an algorithm to calculate a reference image traversal order that ensures correct visibility processing using epipolar geometry [8]. The algorithm is based on epipolar point which is the projection of the viewpoint of a novel view onto the reference image. This epipole divides the reference image's domain into sheets. The warping order for each sheet can be determined using the type of epipolar point (positive or negative). If the epipole is positive, then the traversal must move from the edge of the image towards the epipole. Otherwise for negative epipole, the traversal must move from the epipole towards the edge of the image.

\section{PROPOSED FraMeWORK For MOBILE DEVICES}

In this section, we propose a framework that uses wavelet-based warping technique to render novel views of large images on mobile/ handheld devices. The proposed framework is based on 3D image warping technique. Further, it makes use of restructured warping order cases and scan line coherency proposed by Walia and Verma [9] and Haar wavelet transform to decompose large images. For a level-one transform, this creates four sub images (one approximation and three details). However, we ignore the three detail images and simply warp the approximation image. This reduces image size to one half to its original size along the width as well as height. Similarly, the depth image is also reduced to one half to its original size along the width as well as height by using the Haar wavelet transform. This results in making the mapping from reference image to desired image efficient while generating the novel views, as the rendering time of the warping technique is directly proportional to the image size rather than image complexity. In mobile/ handheld devices where hardware resources are limited, this improves the interactivity and performance. Fig. 5 shows the flowchart of the proposed framework.

Fig. 6 summarizes the algorithm of the proposed framework for mobile devices. The input for this framework is reference and disparity image of size $\mathrm{N}^{*} \mathrm{~N}$ with camera parameters (like center of projection for reference view $\mathbf{C}_{\mathbf{r}}$, center of projection for desired view $\mathbf{C}_{\mathbf{d}}$, and Projection Matrix P). The output for this framework is a novel view of the compressed reference image. The novel view is then rendered on the mobile screen. The proposed framework is implemented using Android Development Tools (ADT) version 22.3; which can run on any mobile device that runs 


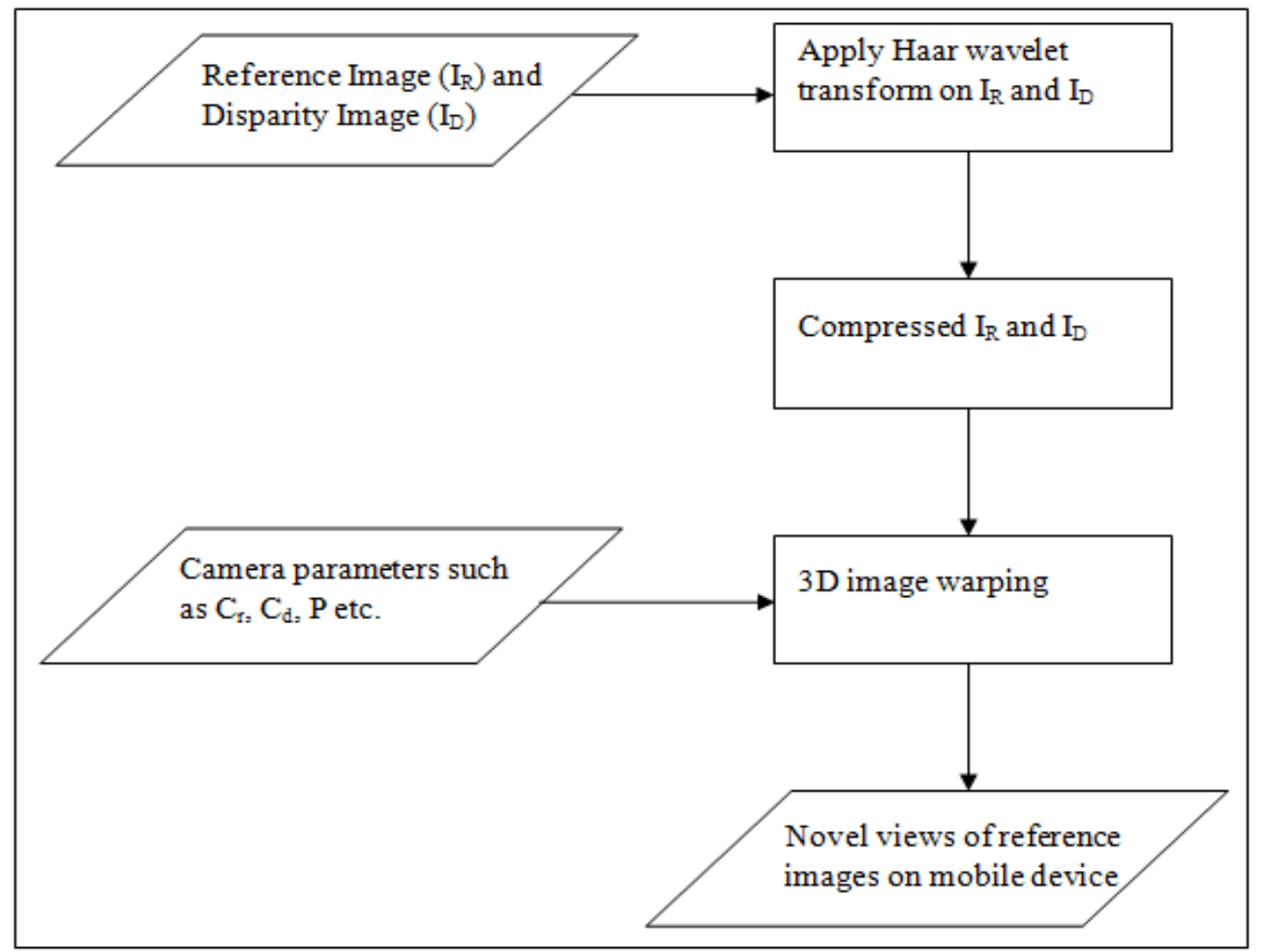

Figure 5. Flowchart of Proposed Framework

on Android OS. User can navigate through the touch screen of the mobile device to change the orientation and position of the camera. Whenever the user performs the navigation, the new values for the camera parameters are computed and the procedure to render the new view is started. The user can also perform zoom-in, zoom-out and reset operations or to change the reference image itself through the DPAD buttons on the mobile device.

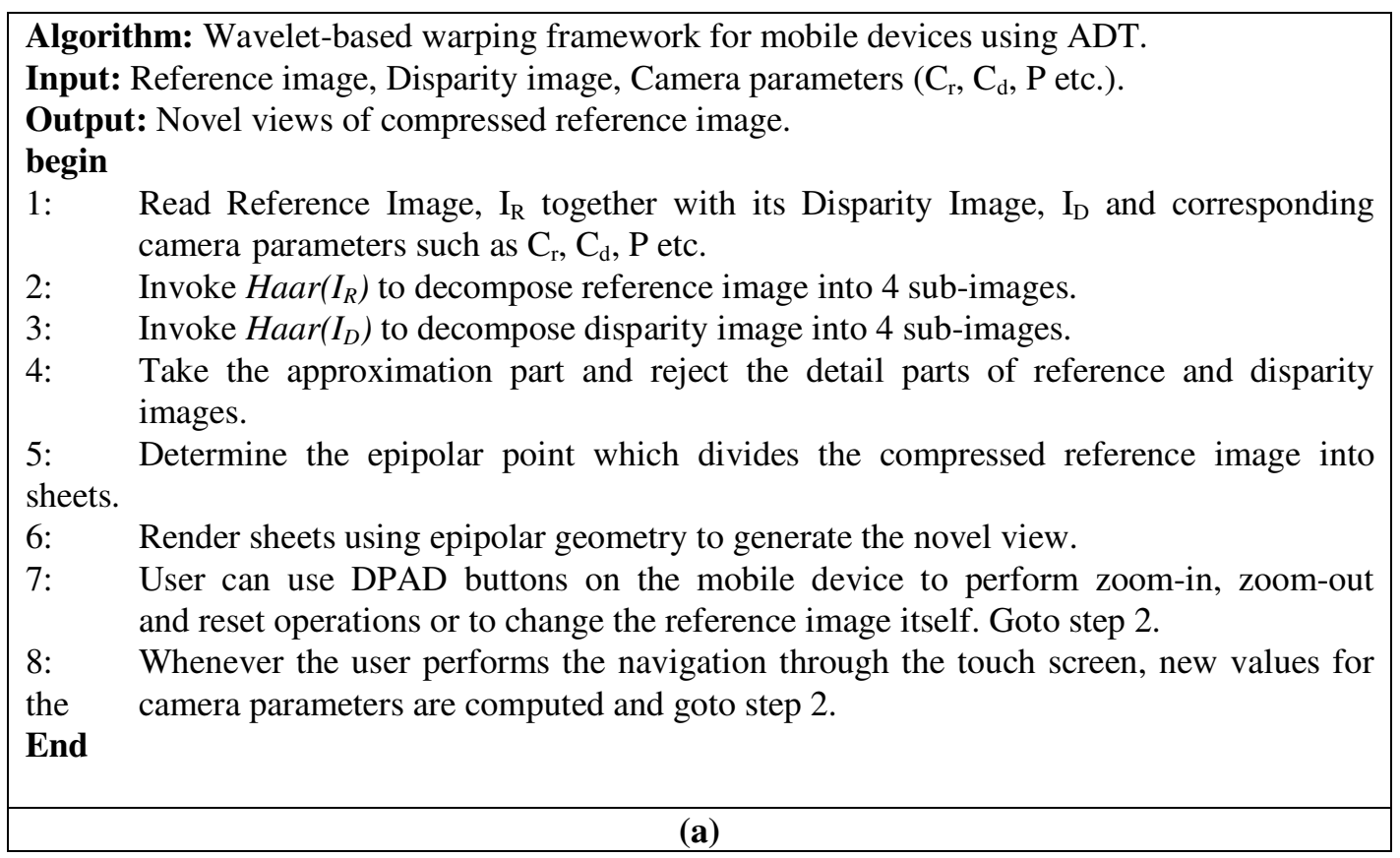




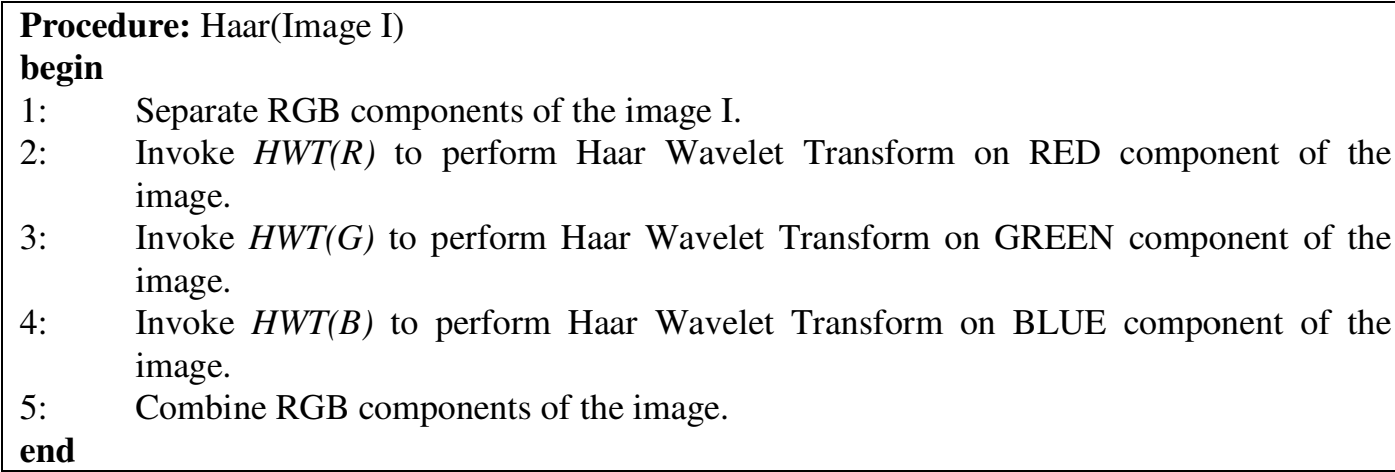

end

Procedure: HWT(Image Component Matrix)

begin

1: $\quad$ For each row in the image matrix:

a) Find the average of each pair of values.

b) Find the difference of each pair of values.

c) Fill the first half with averages.

d) Fill the second half with differences.

e) Select the first half and repeat the process until it has one element.

2: $\quad$ For each column in the image matrix:

a) Find the average of each pair of values.

b) Find the difference of each pair of values.

c) Fill the first half with averages.

d) Fill the second half with differences.

e) Select the first half and repeat the process until it has one element.

3: $\quad$ This produces the updated image matrix containing approximation and detail parts.

end

(c)

Figure 6. Proposed Framework (a) Complete algorithm (b) Procedure Haar (c) Procedure HWT

\section{EXPERIMENTAL RESULTS AND DISCUSSION}

The proposed framework has been implemented using Java Platform Standard Edition 1.6 (update 21) and Android Development Tools (ADT) version 22.3. The experiments have been conducted using Android Virtual Device (AVD) emulator on a machine having $2.5 \mathrm{GHz}$ Intel(R) Core(TM) i5 CPU and 4.0 GB RAM. A set of images taken from a dataset of images [10] has been used in our experiments. Fig. 7 shows a subset of images along with their gray scale images (having depth information).

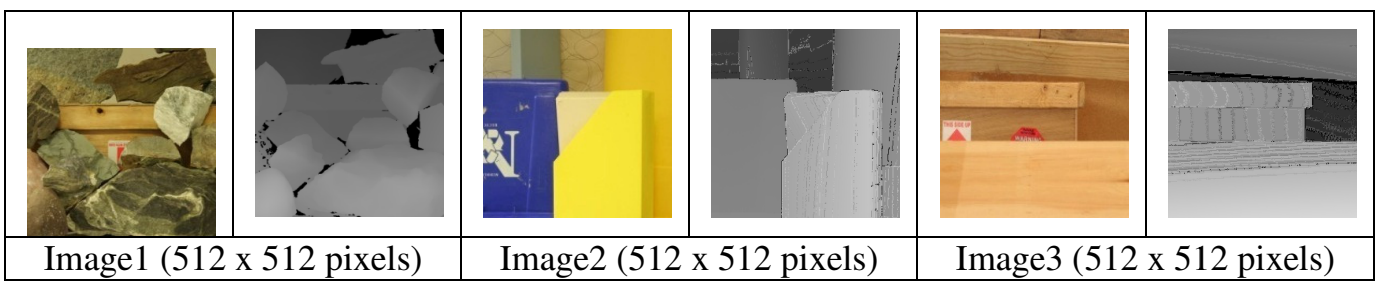

Figure 7. Images (with their depth information) 
In the setup discussed above, experiments have been conducted to evaluate the performance of proposed framework in Android environment on a set of images shown in Fig. 7. The ADT can be used to define AVD (Android Virtual Device) emulators that enable us to simulate the mobile environment on a PC. Fig. 8 shows the output of the proposed rendering framework in the AVD emulator having screen size 3.2" with 512 MB RAM.

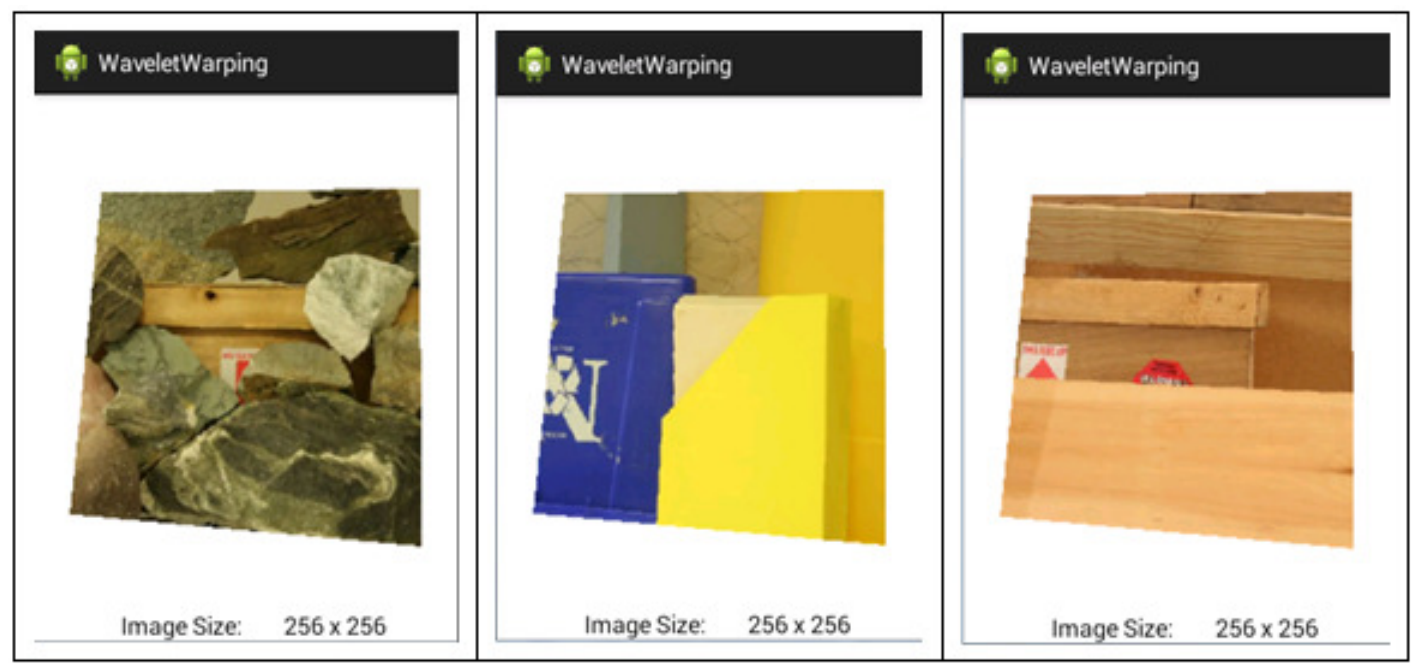

Figure 8. Rendering using proposed framework in AVD emulator

Table 1 gives the rendering times (in milliseconds) of the different images using the proposed framework and its comparison with the warping framework proposed by [9] using ADT. The experimental results show that the proposed framework gives better results for compressed images in terms of rendering time. Further as shown in Fig. 8, the compressed rebuilt image is close to the original image.

Table 1. Rendering time comparison of images shown in Fig. 6

\begin{tabular}{|c|c|c|}
\hline Images & $\begin{array}{c}\text { Rendering time using warping } \\
\text { framework [9] (in ms) }\end{array}$ & $\begin{array}{c}\text { Rendering time using wavelet- } \\
\text { based warping framework (in ms) }\end{array}$ \\
\hline Image1 & 3490 & 2278 \\
\hline Image2 & 3454 & 2293 \\
\hline Image3 & 3462 & 2232 \\
\hline
\end{tabular}

\section{CONCLUSION}

In this paper we propose a wavelet-based warping framework to render novel views of a reference image on mobile devices. By applying the Haar wavelet transform we represent the reference and disparity images in terms of low-resolution images and a set of detail coefficients. By ignoring the detail coefficients and simply warping the approximation image we get the novel view of the reference image. As the rendering time of the warping technique is directly proportional to the image size rather than image complexity, this improves the rendering time. The framework is implemented with Android Development Tools (ADT) and its performance is evaluated. The experimental results show the proposed framework gives better results for compressed images in terms of rendering time. Further, the compressed rebuilt image is close to the original image. 


\section{REFERENCES}

[1] M. Vetterli and J. Kovacevic, "Wavelet and Subband Coding", Prentice Hall PTR, Englewood Cliffs, NJ, 2007.

[2] R. S. Stankovic and B. J. Falkowski, "The Haar wavelet transform: its status and achievements", Computers and Electrical Engineering, Vol.29, No.1, January 2003, pp. 25-44.

[3] Eric J. Stollnitz, Tony D. DeRose and David H. Salesin, "Wavelets for Computer Graphics: A Primer Part 1", IEEE Computer Graphics and Applications, May 1995.

[4] N. Ahmed, T. Natarajan, and K. R. Rao, "Discrete Cosine Transform", IEEE Trans. Computers, vol. C-23, Jan. 1974.

[5] A. Cohen, I. Daubechies and J. C. Feauveau, "Biorthogonal bases of compactly supported wavelets", Communications on Pure and Applied Mathematics, Vol. 45, No. 5, 1992, pp. 485-560.

[6] I. Daubechies, "Ten lectures on wavelets", Vol. 61 of CBMS-NSF Regional Conference Series in Applied Mathematics. Philadelphia, PA: Society for Industrial and Applied Mathematics (SIAM), 1992.

[7] L. McMillan, "An Image-Based Approach to Three-Dimensional Computer Graphics”, Ph.D. Thesis, Department of Computer Science, University of North Carolina at Chapel Hill, 1997.

[8] L. McMillan. “Computing Visibility Without Depth”. Computer Science Technical Report TR95-047, University of North Carolina, Chapel Hill, 1995.

[9] E. Walia and V. Verma, "A Computationally Efficient Framework for 3D Warping Technique", International Journal of Computer Graphics, Vol. 3, No. 1, May 2012, pp.1-10.

[10] Middlebury Stereo Datasets. http://vision.middlebury.edu/stereo/data/ scenes2006.

\section{AUTHORS}

Ekta Walia received her Bachelors degree in Computer Science from Kurukshetra University, India and Masters in Computer Applications as well as Ph.D. (Computer Science) from Punjabi University, Patiala, India respectively. After starting her professional career as a software consultant with DCM DataSystems, New Delhi, India, in 1998, she served as faculty member in the National Institute of Technical Teachers Training and Research (NITTTR), Chandigarh, India for 07 years. From 2007 to 2011, she served in various academic institutes. In July 2011, she joined the Department of Computer Science in South Asian University, New Delhi, where she has been serving as

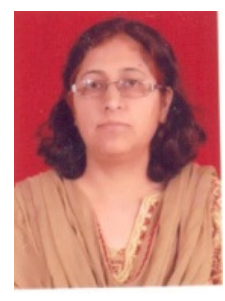
Associate Professor \& Chairperson. Her research interests include 3D Rendering, Digital Image Watermarking, Content Based Image Retrieval and Face Recognition. She has a number of international journal and conference publications in these areas. She has been on the reviewing board of many reputed image processing journals and conferences. She has also chaired sessions in International Conferences of repute.

Vishal Verma is an Assistant Professor at Department of Computer Science, M. L. N. College, Yamuna Nagar, Haryana (INDIA). He is having Masters in Computer Applications from Kurukshetra University, Kurukshetra (INDIA) and M. Phil. (Computer Science) from Madurai Kamaraj University, Madurai (INDIA). His total teaching experience is more than 12 years and is presently pursuing Ph.D. (Computer Science) at Maharishi Markandeshwar University, Mullana, Ambala (INDIA). His current research focus is on Rendering Techniques and Image Processing. He has a number of International journal and conference papers to his credit.

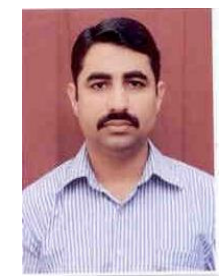

